\title{
Ileocolonic intussusception caused by epithelioid leiomyosarcoma of the ileum: a report of case and review of the literature
}

\author{
Han-Gil Kim ${ }^{1}$, Jung Wook Yang ${ }^{2}$, Soon-Chan Hong ${ }^{1}$, Young-Tae Ju${ }^{1}$, Chi-Young Jeong ${ }^{1}$, Ju-Yeon Kim ${ }^{1}$, \\ Ji-Ho Park ${ }^{1}$, Jin-Kwon Lee ${ }^{3}$, Jae-Myung Kim ${ }^{1}$, Jin-Kyu Cho ${ }^{1}$, Seung-Jin Kwag ${ }^{1}$ \\ Departments of ${ }^{1}$ General Surgery and ${ }^{2}$ Pathology, Gyeongsang National University Hospital, Gyeongsang National University School of \\ Medicine, Jinju; ${ }^{3}$ Department of General Surgery, Gyeongsang National University Changwon Hospital, Gyeongsang National University \\ School of Medicine, Changwon, Korea
}

Small intestinal malignant tumor accounts for about $3 \%$ of all malignant tumors in the gastrointestinal tract, among which $13 \%$ are leiomyosarcoma (LMS). In addition, epithelioid LMS is of very rare occurrence. As small intestinal malignant tumors are initially asymptomatic and nonspecific, diagnosis is often delayed, and this can lead to large tumor at the time of detection and lead to intussusception. We observed ileocolonic intussusception in an 80-year-old male patient who was admitted to the hospital with a complaint of abdominal pain and palpable mass on right lower quadrant. The laparoscopic ileocecectomy was performed by the emergency operation because of obstruction. The pathologic examination revealed that the epithelioid LMS developed in the terminal ileum was the leading point of intussusception. To the best of our knowledge, laparoscopic surgery for ileocolonic intussusception with epithelioid LMS has not yet been reported.

Keywords: Ileal neoplasms; Intussusception; Leiomyosarcoma

\section{INTRODUCTION}

Intussusception is known as the most common cause of intestinal obstruction in children under 2 years of age, and is the second most common cause of emergency surgery in children, after acute appendicitis [1]. However, about 5\% of all intussusception occurs in adults [2]. Unlike pediatric cases, adult intussusception has a leading point or a specific cause in about $80 \%$ [3] and about $2 / 3$ of them can be found in the small intestine. Most of these cases are caused by benign tumors such as small intestinal lipoma or an ad-

Received: Sep 9, 2020 - Revised: Nov 20, 2020 - Accepted: Dec 8, 2020 Correspondence to: Seung-Jin Kwag, M.D.

Department of General Surgery, Gyeongsang National University Hospital, Gyeongsang National University School of Medicine, 79 Gangnam-ro, Jinju 52727, Korea

Tel: +82-55-750-8091, Fax: +82-55-750-9244

E-mail:drksj77@naver.com

ORCID: https://orcid.org/0000-0002-9267-9158

(C) 2022 The Korean Society of Coloproctology

This is an open-access article distributed under the terms of the Creative Commons Attribution NonCommercial License (https://creativecommons.org/licenses/by-nc/4.0) which permits unrestricted noncommercial use, distribution, and reproduction in any medium, provided the original work is properly cited. enomatous polyp, and rarely caused by small intestinal malignant tumor in adults [2].

Small intestinal malignant tumors are of rare occurrence and constitute less than 3\% of all gastrointestinal (GI) tract malignant tumors [4]. Histologically, small intestinal tumors are of different subtypes, with adenocarcinoma being the most frequent (47\%) followed by carcinoid tumors (28\%), leiomyosarcoma (13\%), and lymphomas (12\%) [5]. Most of them are asymptomatic or initial symptoms are nonspecific. Diagnosis of malignant tumors is often delayed, and it takes about 6 to 9 months to diagnose after symptoms appear, making early diagnosis a difficult task [6]. We report a case of ileocecal intussusception on abdominal computed tomography of an 80-year-old man with right lower abdominal pain. After surgical resection, ileal epithelioid leiomyosarcoma was diagnosed. To the best of our knowledge, this is the first report about a case of ileocolonic intussusception caused by epithelioid leiomyosarcoma of the ileum. This study was approved by the Institutional Review Board of Gyeongsang National University Hospital (GNUH 2019-01-024-001), and the informed consent was obtained. 
Volume 38, Number 2, 2022

Ann Coloproctol 2022;38(2):176-180

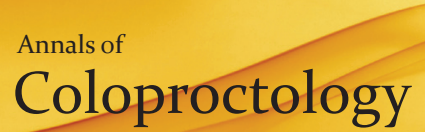

\section{CASE REPORT}

An 80-year-old male patient visited the hospital with a complaint of abdominal pain on right lower quadrant which occurred 3 days ago. Ten months ago, he was diagnosed with non-small cell lung cancer and received radiation therapy. He was treated with a gamma knife 2 months ago for bleeding from brain metastases. Clinical examination demonstrated a tender and palpable mass in the right lower quadrant. He was hemodynamically stable. Detailed labora- tory studies showed all results within normal limits, including complete peripheral blood cell counts and blood biochemistry. However, erythrocyte sedimentation rate was $69 \mathrm{~mm} / \mathrm{hr}$ and Creactive protein was $24.3 \mathrm{mg} / \mathrm{L}$. About $5.5 \times 4.0-\mathrm{cm}$ low-density mass in the terminal ileum and ileocolonic intussusception was observed on the computed tomography scan (Fig. 1).

He underwent an emergency operation using a laparoscopic technique on the same day and ileocolonic type of intussusception was confirmed based on the examination of the abdominal cavity with
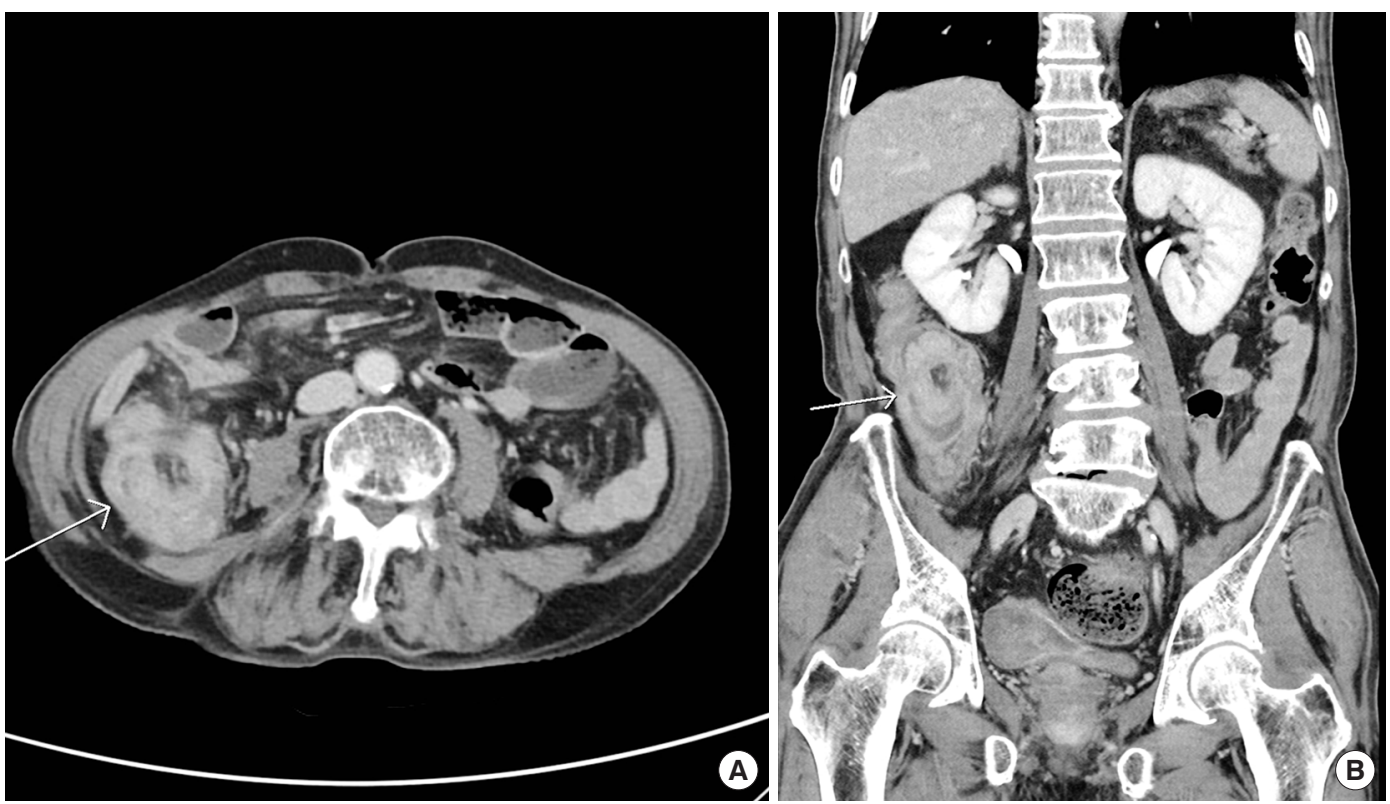

Fig. 1. Abdominal computed tomography of abdomen and pelvis with contrast. An ileocolic intussusception is present (arrows) with the terminal ileum telescoping into the ascending colon. (A) Axial view, (B) coronal view.

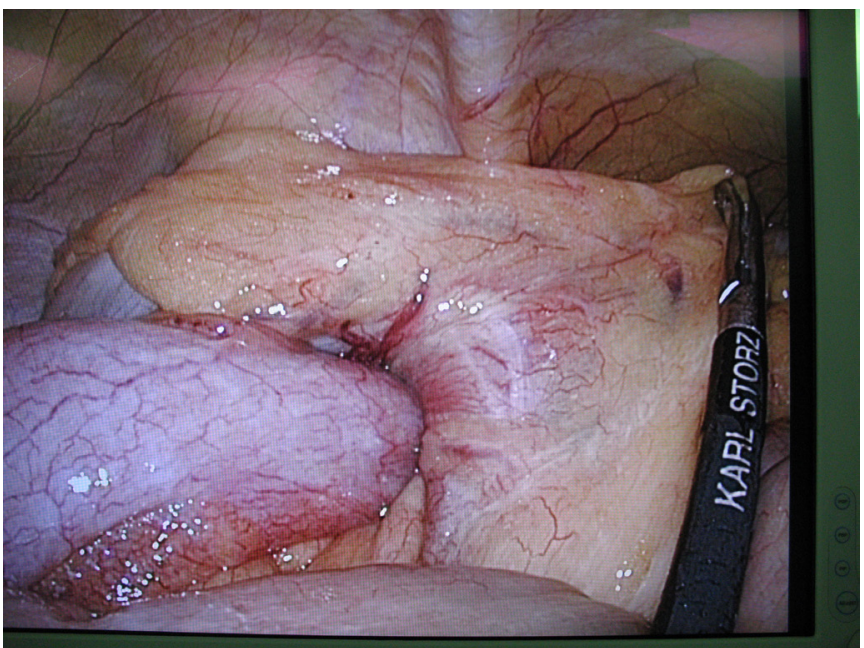

Fig. 2. Intussusception of the ileum into the cecum is seen on the laparoscopic screen.

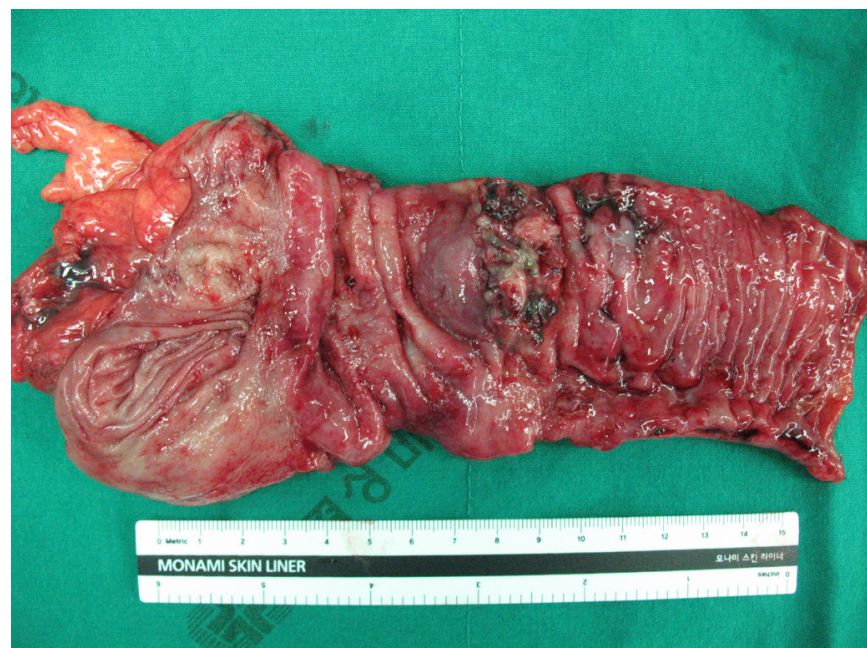

Fig. 3. Gross finding of a $3.5 \times 2.5-\mathrm{cm}$ sized tumor in terminal ileum. 


\section{$\begin{array}{rlr}\text { Annals of } & \text { review of the litera } \\ \text { Coloproctology } & \text { Han-Gil Kim, et al. }\end{array}$}

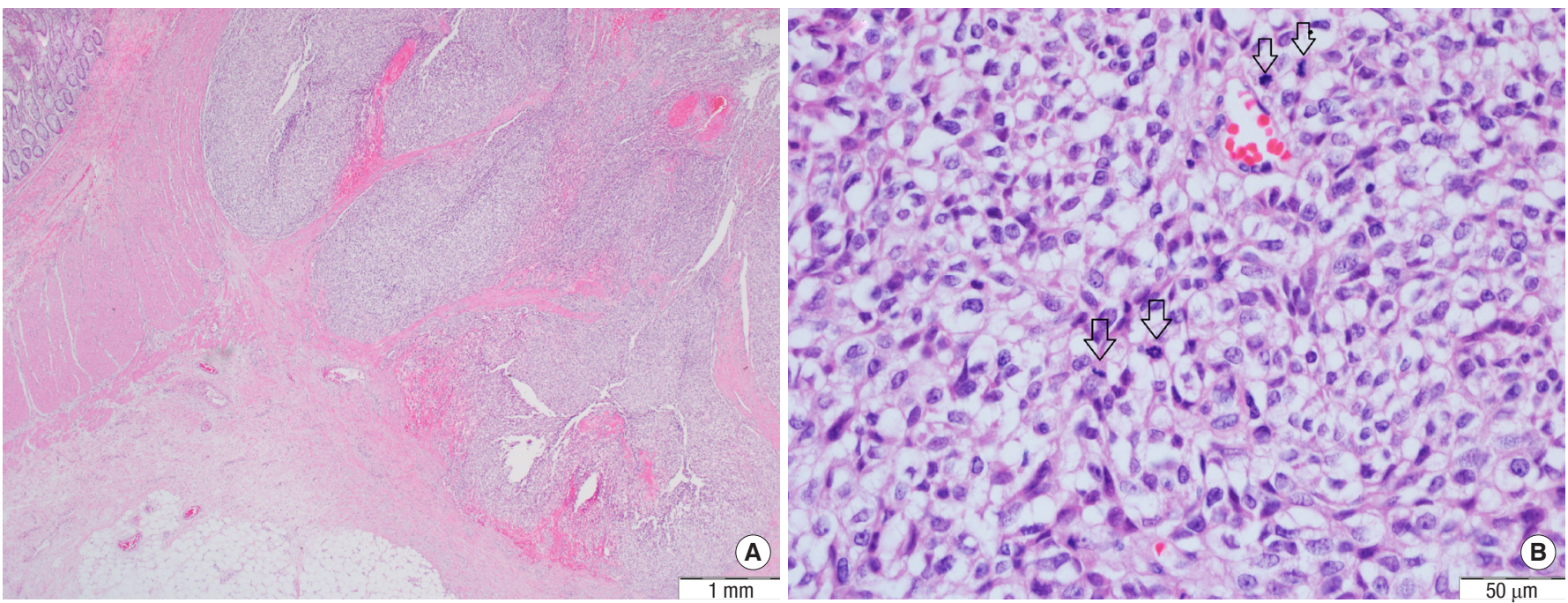

Fig. 4. Microscopic findings of epithelioid leiomyosarcoma. (A) The tumor shows lobular growth pattern and extension proper muscle and subserosa $(\mathrm{H} \& \mathrm{E}, \times 20)$. (B) The tumor cells have eccentric round-to-ovoid vesicular nuclei with inconspicuous nucleoli and abundant vacuolar to lightly eosinophilic cytoplasm. Numerous mitotic figures (arrows) are found up to 40 per 10 high-power fields (H\&E, $\times 400)$.

a camera (Fig. 2). Laparoscopic-assisted ileocecal resection was performed for the treatment of lesions. In the excised colonic specimen, around $4 \times 3 \mathrm{~cm}$ of ulcerofungating mass was observed (Fig. 3). Microscopic examination revealed the tumor as epithelioid leiomyosarcoma with extension to subserosa and 40 mitoses per 10 high-power fields (Fig. 4). Immunohistochemistry of the tumor cells revealed the presence of vimentin and smooth muscle actin (SMA). Among the other markers, CD117 showed a weak positive, but desmin, CD117, discovered on GI stromal tumor 1 (DOG-1), S-100, human melanoma black (HMB)-45, and calponin were negative (Fig. 5). In addition, CK7, CK20, epithelial membrane antigen (EMA), and leukocyte common antigen (LCA) were also negative and not included in the figure. The proximal and distal resection margins measured 10.5 and $15 \mathrm{~cm}$, respectively. No metastasis was found in any of the 14 removed lymph nodes. He was discharged without complications after surgery but died 3 months after surgery from a worsening non-small cell lung cancer.

\section{DISCUSSION}

Small intestinal malignant tumors are rare and comprise less than $3 \%$ of all primary GI neoplasms [4]. There are several conditions that increase the risk of development of small intestinal malignancies, including inflammatory bowel diseases such as Crohn disease, polyposis syndrome, and hereditary nonpolyposis colorectal cancer [7].

Leiomyosarcoma arising in the GI tract accounts for $1 \%$ to $2 \%$ of GI malignancies and the distribution of the lesions is $50 \%$ in the stomach, $30 \%$ in the small intestine, $15 \%$ in the colon, and 5\% in the esophagus [8]. Leiomyosarcoma of the small intestine oc- curs in the order of the jejunum, the ileum, and the duodenum [9].

Leiomyosarcoma is histologically composed of spindle cells with moderate or more differentiation but epithelial cells or a mixture of these 2 types of cells may appear. Epithelioid leiomyosarcoma is characterized by round to polygonal cells with abundant eosinophilic or clear cytoplasm. In the present case, eccentric round-toovoid vesicular nuclei with inconspicuous nucleoli were detected in the pathologic examination [10]. Additional immunohistochemistry tests were used to confirm the diagnosis.

Differential diagnosis which shows similar histologic finding with this case includes epithelioid leiomyoma (positive for SMA and usually negative for desmin), epithelioid GI stromal tumor (positive for CD117 and DOG-1), perivascular epithelioid cell tumor (positive for SMA, S-100, and HMB-45), clear cell sarcoma (positive for S-100 and HMB-45), and malignant glomus tumor (positive for SMA and calponin). Although not shown in the figure, additionally, CK7, CK20, EMA, and LCA were negative. Considering the results of immunohistochemical stains, the diagnosis was consistent with epithelioid leiomyosarcoma.

The clinical features of small intestinal malignant tumors are unfortunately very nonspecific. The patient may have abdominal pain, nausea and/or distension. In the early stages, these symptoms can be misdiagnosed as other benign diseases such as functional GI impairment, which can delay the diagnosis [11]. Because the clinical symptoms are nonspecific, a high index of suspicion is needed to diagnose small intestinal malignant tumors. In later stages, iron deficiency anemia or GI bleeding may be accompanied, but in most of the cases, the amount of hemorrhage is low and the diagnosis is made based on fecal occult blood test rather than by hematochezia $[11,12]$. Unfortunately, the presence of obvious symptoms re- 
Volume 38, Number 2, 2022

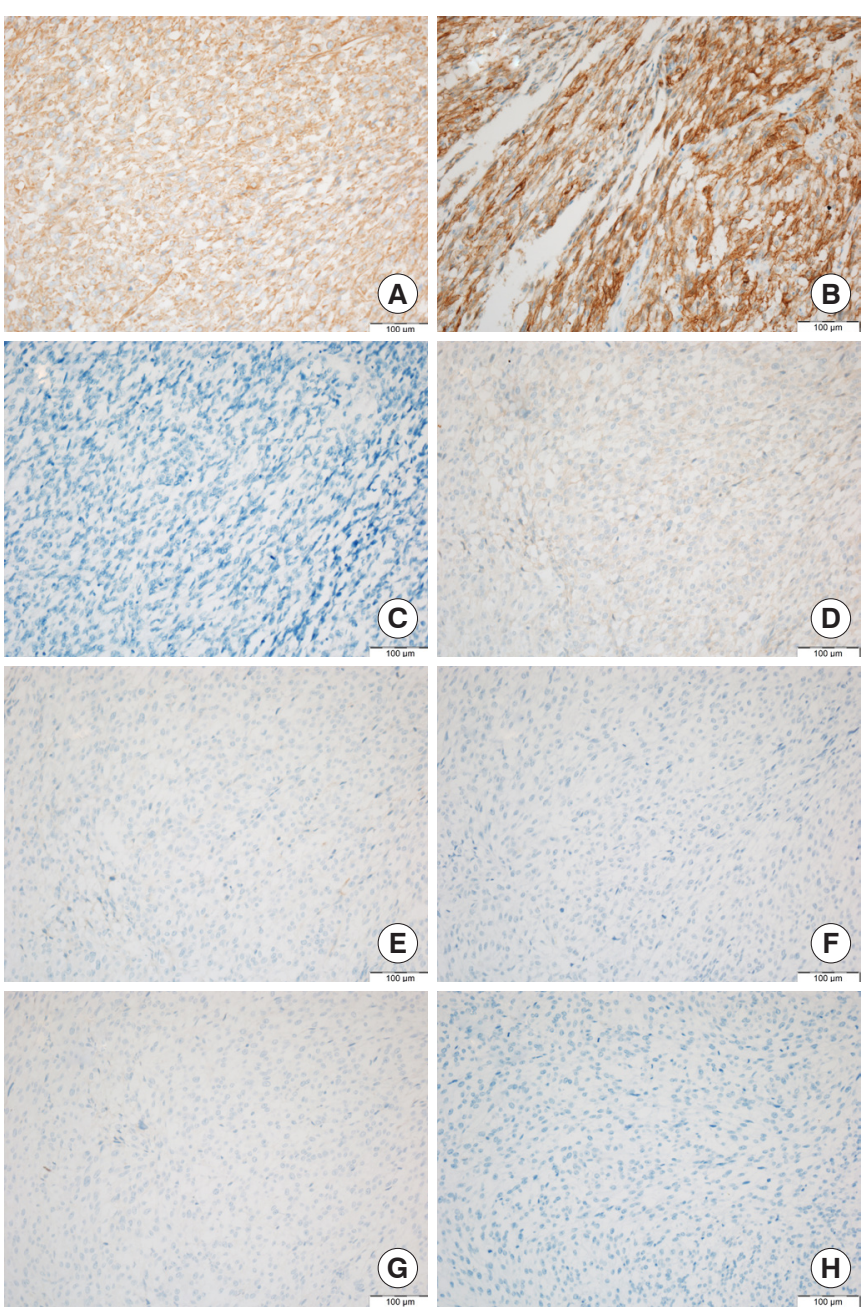

Fig. 5. Immunohistochemistry of epithelioid leiomyosarcoma. The tumor cells express (A) vimentin and (B) smooth muscle actin. The other markers, (C) desmin, (D) CD117, (E) DOG-1, (F) S-100, (G) $\mathrm{HMB}-45$, and $(\mathrm{H})$ calponin are weak positive (only for CD117) or negative $(\times 200)$. DOG-1, discovered on gastrointestinal stromal tumor 1; HMB-45, human melanoma black 45 .

veals local invasion or metastasis, and prognosis may be worse [13].

The only effective treatment of leiomyosarcoma in the small intestine is surgical resection, which is known to require extensive resection including the mesentery, and radiation therapy for leiomyosarcoma of the small intestine has yet to provide clear evidence. It is also known that chemotherapy has a very low response. The clinical differences between leiomyosarcoma and epithelioid leiomyosarcoma in the conventional small intestine are very rare in the latter case, so they are not clearly known.

In the present case, the patient presented with right lower quadrant abdominal pain which led to the detection of a tumor in the terminal ileum. An ileocecectomy was performed using a laparoscope based on the diagnosis of ileocolonic type intussusception.
Pathological examination of the small intestine after surgery revealed the presence of epithelial leiomyosarcoma. In the past, no cases of laparoscopic ileocecectomy with ileocolonic intussusception due to small intestinal epithelial leiomyosarcoma have been reported. However, the PubMed database search presented a case of segmental laparoscopic resection of the small intestine due to leiomyosarcoma described by Hamm et al. in 2013 [14]. One case of laparoscopic resection with ileocolonic intussusception caused by ileal leiomyosarcoma described by Guzel et al. in 2016 [7] was evident, although it was not a case of epithelioid leiomyosarcoma.

In conclusion, leiomyosarcoma of the ileum is rare and the symptoms are nonspecific, making it difficult to identify at the first diagnosis. However, in the present case, we experienced a rare case of epithelioid leiomyosarcoma of the ileum, which was found to be the leading point for ileocolonic intussusception.

\section{CONFLICT OF INTEREST}

No potential conflict of interest relevant to this article was reported.

\section{FUNDING}

This study was supported by grants of the Basic Science Research Program through the National Research Foundation of Korea (NRF) funded by the Ministry of Science, ICT and Future Plan ning, Republic of Korea (No. NRF-NRF-2018R1D1A1B07045469).

\section{REFERENCES}

1. Zubaidi A, Al-Saif F, Silverman R. Adult intussusception: a retrospective review. Dis Colon Rectum 2006;49:1546-51.

2. Begos DG, Sandor A, Modlin IM. The diagnosis and management of adult intussusception. Am J Surg 1997;173:88-94.

3. Triantopoulou C, Vassilaki A, Filippou D, Velonakis S, Dervenis C, Koulentianos E. Adult ileocolic intussusception secondary to a submucosal cecal lipoma. Abdom Imaging 2004;29:426-8.

4. Farhat MH, Shamseddine AI, Barada KA. Small bowel tumors: clinical presentation, prognosis, and outcome in 33 patients in a tertiary care center. J Oncol 2008;2008:212067.

5. North JH, Pack MS. Malignant tumors of the small intestine: a review of 144 cases. Am Surg 2000;66:46-51.

6. O'Riordan BG, Vilor M, Herrera L. Small bowel tumors: an overview. Dig Dis 1996;14:245-57.

7. Guzel T, Mech K, Mazurkiewicz M, Dąbrowski B, Lech G, Chaber A, et al. A very rare case of a small bowel leiomyosarcoma leading to ileocaecal intussusception treated with a laparoscopic resection: a case report and a literature review. World J Surg Oncol 2016;14: 48.

8. Conlon KC, Casper ES, Brennan MF. Primary gastrointestinal sarcomas: analysis of prognostic variables. Ann Surg Oncol 1995;2: 26-31.

9. Akwari OE, Dozois RR, Weiland LH, Beahrs OH. Leiomyosarco- 


\section{Coloproctology Han-Gil Kim, et al.}

ma of the small and large bowel. Cancer 1978;42:1375-84.

10. Moinfar F, Azodi M, Tavassoli FA. Uterine sarcomas. Pathology 2007;39:55-71.

11. Islam RS, Leighton JA, Pasha SF. Evaluation and management of small-bowel tumors in the era of deep enteroscopy. Gastrointest Endosc 2014;79:732-40.

12. Paski SC, Semrad CE. Small bowel tumors. Gastrointest Endosc
Clin N Am 2009;19:461-79.

13. Cheung DY, Choi MG. Current advance in small bowel tumors. Clin Endosc 2011;44:13-21.

14. Hamm JK, Chaudhery SI, Kim RH. Laparoscopic resection of small bowel sarcoma. Surg Laparosc Endosc Percutan Tech 2013; 23:e138-40. 